\title{
Correction to: Clozapine-related neutropenia, myocarditis and cardiomyopathy adverse event reports in Australia 1993-2014
}

\author{
Samantha A. Hollingworth ${ }^{1} \cdot$ Karl Winckel $^{1,2} \cdot$ Nargess Saiepour $^{3} \cdot$ Amanda J. Wheeler $^{4,5} \cdot$ Nicholas Myles $^{6}$. \\ Dan Siskind ${ }^{7,8}$
}

Published online: 23 April 2018

(C) Springer-Verlag GmbH Germany, part of Springer Nature 2018

\section{Correction to: Psychopharmacology \\ https://doi.org/10.1007/s00213-018-4881-0}

The authors would like to change the statement found in the results section of the abstract from "There were five reported deaths from neutropenia and cardiomyopathy." to "There were five, 13, and two reported deaths from neutropenia, myocarditis, and cardiomyopathy, respectively."

The online version of the original article can be found at https://oi.org/ $10.1007 / \mathrm{s} 00213-018-4881-0$

Samantha A. Hollingworth

s.hollingworth@uq.edu.au

Karl Winckel

k.winckel@pharmacy.uq.edu.au

Nargess Saiepour

n.saiepour@uq.edu.au

Amanda J. Wheeler

a.wheeler@griffith.edu.au

Nicholas Myles

nicholas_myles@hotmail.com

Dan Siskind

d.siskind@uq.edu.au

1 School of Pharmacy, The University of Queensland, 20 Cornwall St, Woolloongabba, QLD 4102, Australia
2 Princess Alexandra Hospital, 199 Ipswich Rd, Woolloongabba, QLD 4102, Australia

3 School of Public Health, The University of Queensland, Herston Rd, Herston, QLD 4006, Australia

4 Menzies Health Institute Queensland, Meadowbrook, QLD 4103, Australia

5 Faculty of Medical and Health Sciences, University of Auckland, 85 Park Road, Grafton, New Zealand

6 SA Pathology, PO Box 14 Rundle Mall, Adelaide, SA 5000, Australia

7 Metro South Addiction and Mental Health Service, 228 Logan Road, Woolloongabba, QLD 4102, Australia

8 School of Medicine, The University of Queensland, Herston Rd, Herston, QLD 4006, Australia 